\title{
Le projet de restauration de Bordj Istanbul, à Alger
}

\author{
Restoration of the Bordj Istanbul fort in Algiers
}

\section{Ouassila Menouer}

Institut d'Architecture et Urbanisme - Laboratoire Environnement et Technologie pour l'Architecture et le Patrimoine - Université Saâd Dahleb, Blida 1, Algeria, menouerouassila@yahoo.fr

\begin{abstract}
Throughout history, Algerian coastal towns have been subject to various threats by sea, which has resulted in different types of fortification works. Their variety depended on their period of construction, the materials used and the genius of the place. In Algiers, these structures of fortifications vary between fortified castle, ramparts, watchtowers, etc. However, this historical legacy is, day after day, threatened with disappearance for lack of support. The Bordj Istanbul, or Turkish fort, is one of the most important, in Algiers. Before the launch of its restoration, the monument was in a state of advanced degradation caused by abandonment, vandalism and the aggression of the sea. In 2012, the operation was started by sorting the dislocated stone blocks of the building and consolidating its structure. The project has been reflected in a scientific approach that pays particular attention to the aesthetic integrity of the monument. The absence of archival documents has been surpassed by using comparative readings of the same type of forts and the stratigraphic study of the monument. His defensive function being over, it was necessary to think about his conversion. The character of the place, the architectural aspect of the building and some alterations of its material has aroused the idea of the development of the monument in a gastronomic restaurant by the sea within a private beach. Today, works have been closed and the opening of the restaurant is planned for the next summer season.
\end{abstract}

Keywords: Restoration, fort Bordj Istanbul, conservation, architectural heritage.

\section{Introduction}

Sur le littoral d'Alger, au environ de Bordj ElKiffan, se trouve un petit joyau architectural quasiment inaperçu, le fort Bordj Istanbul, appelé, également, Bordj Eturc. L'édifice militaire fait partie de la série des fortins qui jalonnaient la baie d'Alger. Il témoigne de l'architecture militaire du XIX ${ }^{\mathrm{ème}}$ siècle, importée par les français en Algérie, à cette époque (Fig. 1).

Une inscription gravée sur l'encadrement de sa porte d'accès atteste que le fort a été construit en 1861. Il fut classé dans la $2^{\text {ème }}$ série des postes militaires par décret ministériel. Son assiette de quatre hectares fut acquise par expropriation pour utilité publique. Selon des témoignages, le fort était délimité par un fossé d'enceinte et jouissait d'un puit et d'un abreuvoir. Les traces de ces derniers ont été mises au jour lors des travaux de restauration que le monument a connu récemment.

En effet, le chantier de restauration du fort Bordj Istanbul a été lancé en 2012. L'édifice se trouvait dans un état de dégradation assez avancé, une dégradation marquée surtout par la perte de sa matière physique en différents endroits, à l'instar des encadrements de ses meurtrières, du parapete de sa terrasse, des voûtes couvrant 


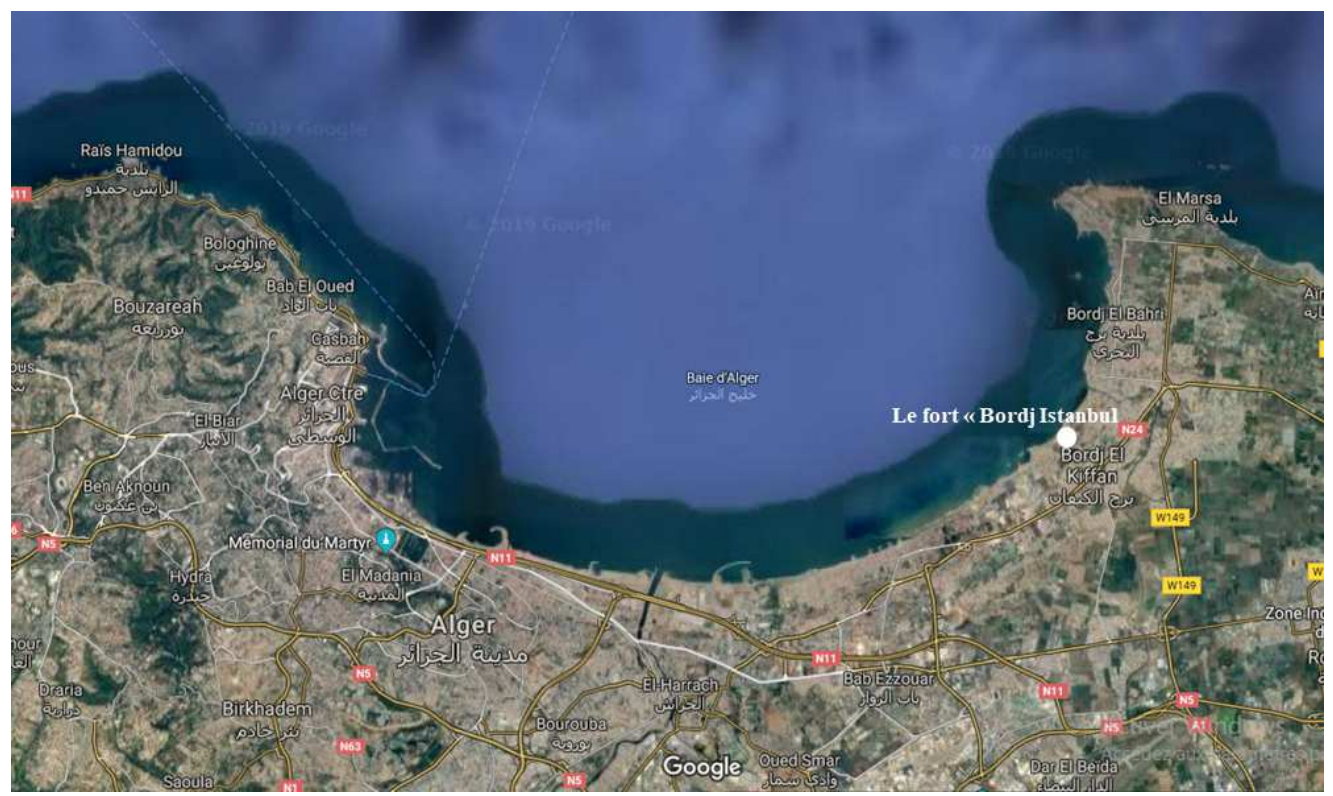

Fig. 1. Situation du fort Bordj Istanbul à Bordj El-Kiffan sur le littoral de la baie d'Alger (Google Earth).

les deux escaliers, etc. Par ailleurs l'abandon et l'exposition du fort aux effets de mer ont augmenté la dégradation de l'édifice.

Les blocs de pierre formant ses structures présentaient une texture alvéolée, sur une profondeur assez importante. Les traces de sel et d'humidité couvraient ses murs, surtout, sur les niveaux du rez-de-fossé et de l'étage. Enfin, la dislocation du chainage d'angle, réalisé en pierre de taille, constituait le désordre le plus le plus important et le plus soucieux.

L'enjeu du projet de restauration du fort Bordj Istanbul résidait dans le fait que ce dernier a été confié à une jeune équipe algérienne qui cherche à s'imposer sur le marché du patrimoine. La difficulté fut augmentée par les courts et les délais octroyés au projet ainsi que par la non disponibilité des documents d'archives pouvant contribuer à la reconnaissance de l'authenticité de l'œuvre.

\section{Identification et description architecturale du monument}

A défaut de documentation historique, le seul moyen d'identifier la typologie architecturale du fort Bordj Istanbul fut le recours à une lecture comparative de l'édifice avec ceux de configuration similaire et érigés à la même période, c'està-dire au XIX ${ }^{\text {ème }}$ siècle. Il s'est avéré qu'il s'agit d'un "corps de garde type 1846", un des modèles de fortification littorale réalisés entre la fin des années 1840 et la fin des années 1860, pour servir de réduits à des batteries de côtes ou des postes garde-côtes (Fig. 2). Dans cette logique, le fort Bordj Istanbul devait servir celui de Bordj El-Kiffan, situé non loin et qui est de fondation ottomane (Boutin, 1927). Ce type de fortification a été érigé, en France, en Corse, en Algérie, au Sénégal et aux Antilles, selon des plans-types arrêtés par le Comité des fortifications, en 1846, (Duvergier, 1847). Il est reconnu comme un corps de garde crénelé $\mathrm{n}^{\circ} 2$ destiné à abriter quarante (40) hommes.

Les corps de garde crénelés $\mathrm{n}^{\circ} 2$ sont des bâtiments qui devaient assurer trois fonctions : la première est d'ordre logistique, c'est-à-dire, abriter les réserves de poudre, le matériel de l'artillerie ainsi que les vivres de la garnison. La deuxième est une fonction de casernement, les chambrées sont destinées aux logements de la troupe. Et enfin, la dernière fonction est la fonction défensive, les créneaux de tir, les bretèches et le pont levis en attestent. 

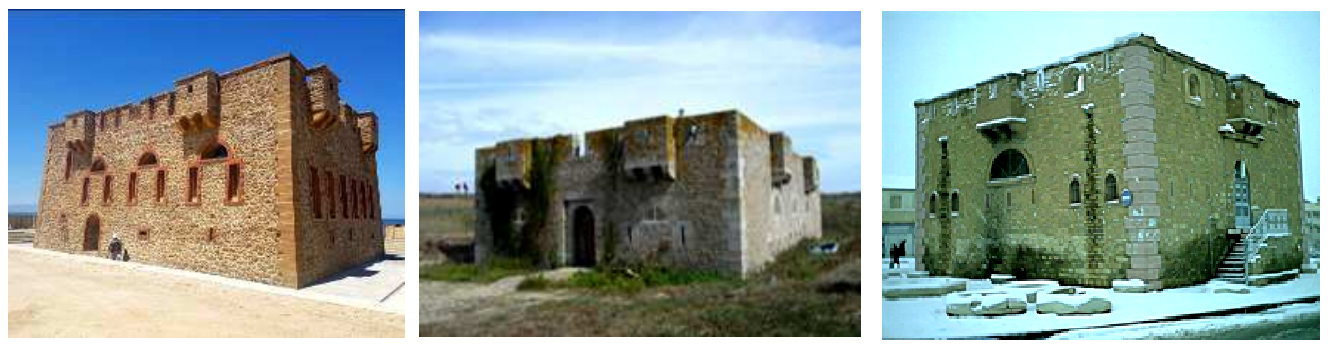

Fig. 2. Le prototype "corps de grade crénelés type 1846". Gauche : Le fort Bordj Istanbul, Alger, Algérie (Auteur, 2012). Centre : Le réduit "type 1846", de Belle-île-en mer, France (http://a54.idata.over-blog.com/0/12/33/28/Belleile-en-Mer/ Belle-ile-454-.jpg). Droite : Le fortin Bruyès, Djelfa, Algérie (https://i0.wp.com/ cciondjelfa.com/ar/wpcontent/uploads/2014/03/djelfa-new-3.jpg?resize=300\%2C225).

Du point de vue organisation spatiale, Bordj Istanbul se développe en trois niveaux : le "rez-defossé" accessible seulement par l'étage, le niveau principal où se trouvent l'entrée avec un pont-levis, le troisième niveau est la terrasse à ciel ouvert munie d'un parapet crénelé.

Les sondages préconisés, lors des travaux préliminaires à la restauration de l'édifice, ont révélé la présence d'un niveau souterrain, il s'agit d'une citerne ayant servie, probablement, à emmagasiner les eaux de pluie recueillies depuis la terrasse.

2.1. Le niveau du rez-de-fossé est organisé en quatre travées, trois d'entre-elles sont recouvertes de voûtes en berceau et la quatrième subdivisée en trois espaces, aussi, voûtés.

Deux escaliers en maçonnerie, menant aux étages supérieurs (le niveau principal et la terrasse), sont aménagés aux extrémités de la travée centrale. Des ouvertures en forme de meurtrières sont aménagées dans les murs périphériques lesquels réalisés en blocs de pierre dans une épaisseur de $50 \mathrm{~cm}$ environ.

\subsection{Le niveau principal ou l'étage est organi-} sé, à son tour, en quatre travées voûtées dont celle de l'extrémité divisée en trois espaces. Sur cette dernière (la travée de l'extrémité), s'organisent les deux escaliers reliant les différents niveaux de part et d'autre d'un sas, l'unique accès au fort, précédé d'un un pontlevis afin de traverser le fossé qui a existé et qui a été révélé lors des sondages effectués in situ.

2.3. Le niveau de la terrasse dont l'accès se fait par les deux escaliers reliant les niveaux infé- rieurs. Elle est délimitée par un parapet en forme de garde-corps muni de meurtrières sur toute sa circonférence. Sur chaque façade sont aménagées deux bretèches en forme d'encorbellements.

3. La structure de l'édifice est en murs porteurs réalisés en blocs de pierre de tuf joints en mortier de terre à l'origine. Sauf que, suite aux différentes opérations de réparation auxquelles l'édifice a été sujet, certains pans sont joints en mortier de ciment.

Les chainages d'angles ainsi que l'encadrement de l'entrée principale (en pont-levis) sont réalisés en blocs de pierre de tuf taillée, alors que les encadrements des meurtrières et des fenêtres sont en brique de terre cuite. L'ensemble des espaces du fort (citernes, rez-de-fossé et l'étage) sont couverts en voûtes en berceau réalisées en bloc de pierre bleue (plus dure) joints en mortier de terre.

Contrairement aux autres forts de la baie d'Alger, Bordj Istanbul a échappé, par on ne sait quel miracle, à l'envahissement du béton. Cependant, il reste exposé à différentes formes de dégradation et d'altération, notamment, l'effet de déminéralisation de la pierre des façades, le vandalisme et l'abandon.

Le diagnostic, établi à travers la connaissance approfondie de l'édifice basée sur l'observation directe et les sondages, a permis de produire une documentation graphique (les relevés architecturaux) jusqu'alors inexistante (Fig. 3). Il a permis, également, d'identifier les pathologies que présentait le monument. Ces dernières étaient tant superficielles que structurelles. 


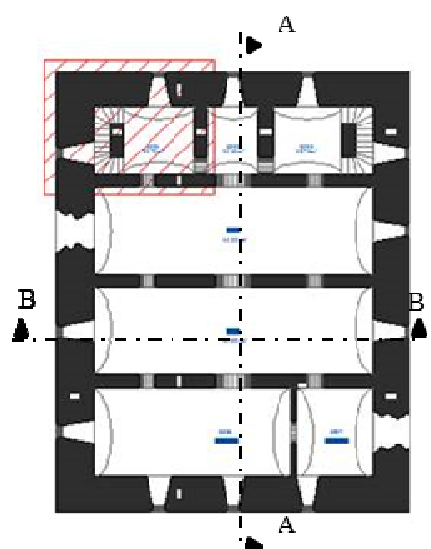

Plan du rez-de-fosse

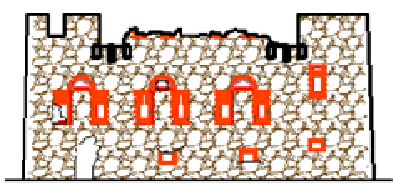

Elévation Nord

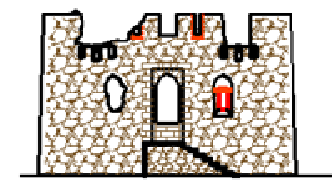

Elévation Onest

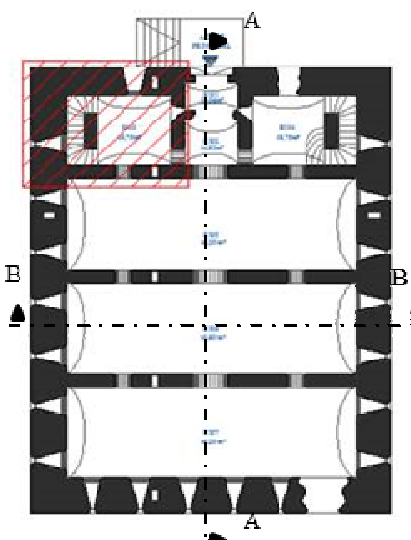

Plan du niveau pifncipal (1'étage)

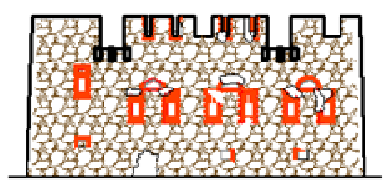

Elévation sud

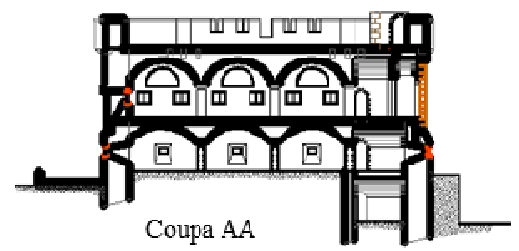

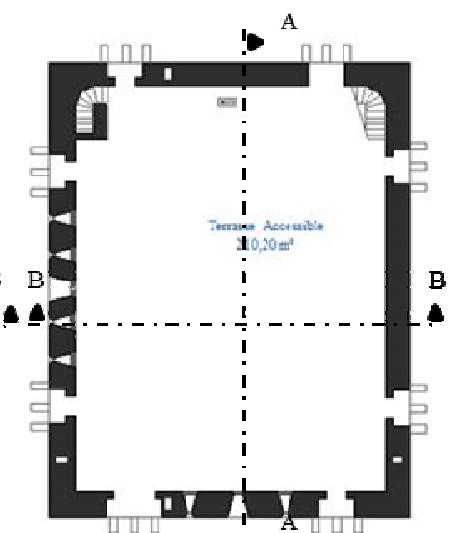

Plande la terrasse

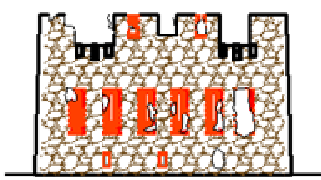

Elevvarion Est

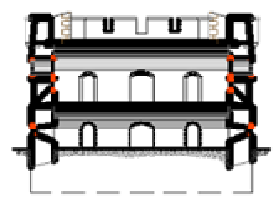

Coupe BB

Fig. 3. Dossier graphique du fort Bordj Istanbul (BET Monart, 2012).

3.1. Les pathologies superficielles telles que des microfissures coplanaires sur les parements des murs, des fissures profondes sur les murs, l'éclatement de la maçonnerie par l'effet des éléments métalliques ancrés dans les murs, l'altération des joints de l'appareillage de la maçonnerie, la déminéralisation de la pierre par l'effet des sels de mer, l'altération des enduits intérieurs, ...

3.2. Les pathologies structurelles au niveau des voûtes en berceau telles que les traces d'humidité due à la remontée capillaire des eaux $\mathrm{du}$ sol et à l'infiltration des eaux de pluie, ce qui a permis l'apparition de la végétation sur les maçonneries, la perte de la matière des mortiers de jointement des murs et des voûtes, la dislocation de l'angle de chainage au niveau de la terrasse, etc. (Fig. 4).

\section{4. Étude stratigraphique ou lecture compara- tive?}

La restitution de l'intégrité matérielle d'une œuvre constitue un des fondements de la restauration des biens culturels (Boito, 2013). Dans le domaine des édifices historiques, une discipline datant des années 90 commence à faire preuve d'efficacité dans l'interprétation des traces matérielles pour la connaissance de l'architecture historique. Il s'agit de l'archéo-logie du bâti, appelée également l'archéologie du mur ou encore l'archéologie du construit, plusieurs appellations convergeant toutes vers une analyse quantitative des objets étudiés, une description détaillée et une interprétation menée sur les observations réalisées, suivant une méthodologie et des outils conceptuels spécifi-ques (Reveyron, 2002). 


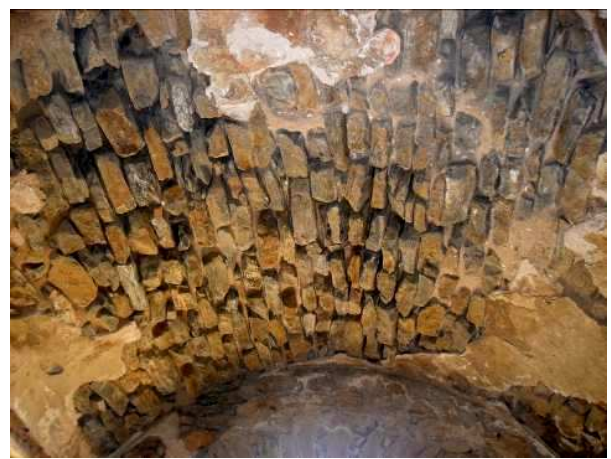

Fig. 4. La maçonnerie des voûtes en berceau (Auteur, 2012).

Dans le cas du fort Bordj Istanbul, l'étude stratigraphique, outil fondamental de l'archéo-logie du bâti, a permis de faire des lectures pertinentes pour la connaissance de l'évolution de la construction de l'édifice, à travers la reconnaissance de :

- la nature des matériaux de construction,

- la technique et la période de construction de l'édifice,

- et des différents altérations et dommages que l'édifice a subis à travers le temps.

Cependant, la restitution de l'image de l'édifice manque de certains détails d'ordre philologique (Brandi, 1977), d'où le recours à un autre outil méthodologique, à savoir la lecture comparative.

En effet, les informations acquises de l'étude stratigraphique ont permis de situer le fort comme étant une batterie défensive datant du XIX $^{\text {ème }}$ siècle et donc érigé par le génie militaire français. Une recherche sur l'architecture militaire française du XIX ${ }^{\text {ème }}$ siècle a permis de reconnaitre d'édifice comme étant un prototype des corps de garde type 1846, construit un peu partout en territoire français de la méditerranée.

Ainsi, il a été possible de restituer l'architecture des façades et replacer l'ensemble des ouvertures, fenêtres et meurtrières, comme il a été possible de situer les lieux de sondage afin de retrouver la citerne, les conduites de descente des eaux de pluie et les puits de lumières. Enfin il a été possible d'établir les dessins des plans et des coupes avec détails et de répondre aux questions soulevées lors de la réalisation des relevés archi- tecturaux, entre autre l'absence du fossé qui est une composante essentielle des édifices militaires isolés ; l'authenticité de l'escalier aménagé à l'extérieur menant à l'étage directement; la présence d'un pont levis et ses éventuels encrages dans les murs; la source ou le puits ayant alimenté le fort en eau douce ;...

\section{Les relevés et les premières idées de mise en valeur du monument}

Les premières intentions projectuelles en matière de restauration et de mise en valeur du fort Bordj Istanbul (confortement, complément des lacunes et aménagement) ont commencé à se formuler lors des premières visites in situ.

L'avantage dans ce projet, est que l'édifice se présentait quasiment dénudé de revêtements (sauf à l'intérieur). Il présentait, également, des pertes de sa matière physique dans des endroits assez intéressants à l'instar de la disparition d'une partie du parapet de la façade donnant sur la mer. La pathologie a engendré même l'idée de la valeur d'usage à lui attribuer.

\subsection{Le confortement des structures}

En s'appuyant sur l'interprétation des informations livrées par les deux outils méthodologiques adoptés, à savoir l'étude stratigraphique et la lecture comparative, le projet de restauration du fort Bordj Istanbul a été réfléchi dans le respect total des principes de la restauration, notamment en matière de distinction entre le nouveau et l'ancien (Charte de Venise, 1964). Parmi les opérations préconisées :

- relier la citerne au niveau du rez-de-fossé par un escalier métallique distinct de la matière authentique du monument,

- compléter l'encadrement des meurtrières en disposant l'appareillage en brique de terre cuite en retrait par rapport à l'existant, afin de distinguer le nouveau de l'ancien,

- restituer la partie disparue du parapet de la terrasse par un barreaudage en verre et aluminium afin de permettre une vue panoramique sur la mer tout en réintégrant l'image de la terrasse ceinturée. 
- restituer la cage de l'escalier, en état de ruine, située dans la terrasse, (Fig. 5),

- consolider l'angle en pierre disloqué par la perte de la matière des joints de mortier en ayant recours à la technique du démontage et du remontage de la maçonnerie: découdre et recoudre (Fig. 6).

- corriger les imperfections de construction et les défauts provoqués par les opérations de transformation et de remaniement que le monument a subi par la réfection des joints de mortier des murs et des voûtes, le colmatage des alvéoles et des perforations de la pierre causées par l'effet des sels de mer, ...

\subsection{Le projet d'architecture : un restaurant gastronomique en bord de mer}

Les qualités spatiales du monument telles que son implantation en bord de mer, dans une plage agréable, la présence d'une terrasse accessible,

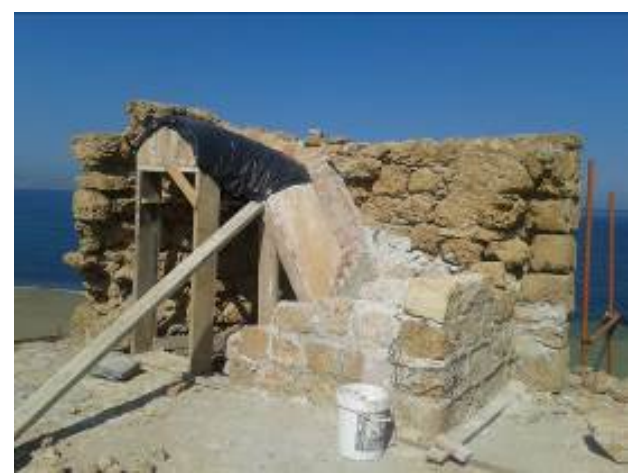

des espaces intérieurs voûtés agréables et invitant à la convivialité, etc., ont favorisé l'idée d'un restaurant assez spécialisé vu l'exiguïté des lieux, d'où le restaurant gastronomique, un lieu ludique cherchant à mettre la gastronomie algéroise à l'honneur : plats de qualité, accueil attentif, service soigné et cadre agréable.

La citerne mis au jour, située au sous-sol et accessible par l'escalier métallique, servira de lieu de stockage des denrées fraiches alors que le niveau du rez-de-fossé sera réservé aux cuisines, aux sanitaires et autres espaces de service.

L'étage, le niveau de l'accès public ainsi que la terrasse seront réservés aux espaces de consommation. L'entrée se fera directement à partir de la plage par un escalier donnant sur un sas servant d'accueil. Ce dernier donne, d'abords, sur deux petites salles de consom-mation puis aboutira sur une plus grande. Les salles de consommation seront abritées dans les espaces voûtés du fort.

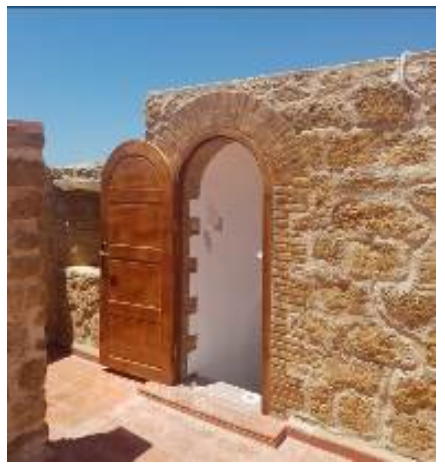

Fig. 5. La cage d'escalier de la terrasse avant et après (Auteur, 2012 et 2016).
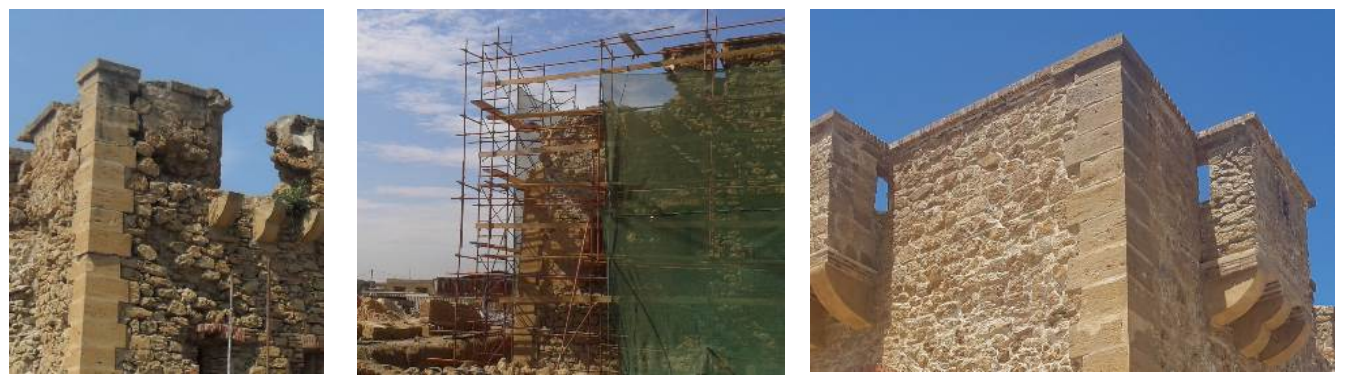

Fig. 6 : L'angle du parapet disloqué, avant en cours et après confortement (Auteur, 2012 et 2016). 
La terrasse abritera un espace de consommation en plein air. Ayant une vue agréable sur la mer, l'espace sera protégé du soleil par des paresoleils, soigneusement, conçus selon l'ameublement et l'aménagement du lieu.

\section{Conclusion}

Quel que soit l'alternative choisie, et en raison de ses qualités spatiales, architecturales et environnementales, l'exploitation du monument, le fort Bordj Istanbul devra être à usage restreint car la surutilisation, le flux touristique intense en période estivale, le manque d'entretien, et d'autres raisons encore constituent les causes principales de dégradation des édifices publiques, en particulier ceux qui sont dotés d'une haute valeur patrimoniale.

Enfin, Bordj Istanbul fait partie des nombreux monuments historiques algériens qui ont connu des opérations de restauration dans un contexte législatif cadré par la loi 98-04 relative à la pro- tection des biens culturels. Le projet a été réfléchi selon une approche scientifique accordant un intérêt particulier à l'intégrité esthétique du monument.

L'absence des documents d'archives a été dépassée en ayant recours aux lectures comparatives des fortins de même typologie et à la lecture stratigraphique de ses structures. Sa fonction défensive étant révolue, il a été nécessaire de penser à une reconversion. Le caractère du lieu, l'aspect architectural de l'édifice ainsi que certaines altérations dans le parapet de sa tressasse ont suscité l'idée de l'aménagement du monument en restaurant gastronomique en bord de mer au sein d'une plage privée, une idée qui a été bien accueillie auprès des autorités concernées.

Aujourd'hui, les travaux de restauration et de mise en valeur sont clôturés et l'ouverture du restaurant est prévue pour la prochaine saison estivale.

\section{Bibliography}

Boito, C. (2013). Conserver ou restaurer? (1893), éd. de l'encyclopédie des nuisances, Saint-Front-sur-Nizonne, France, p. 40.

Boutin, V.Y. (1927). Reconnaissance des villes, forts, et batteries d'Alger par... Boutin (1808) suivie des Mémoires sur Alger par les consuls de Kercy (1791) et Dubois-Thainville (1809), Esquer, G. ed., Librairie ancienne Honoré Champion, Paris, p. 30.

Brandi, C. (1977). Téoria del restauro, Piccola Biblioteca Einaudi Ed., Torino, p. 6.

ICOMOS. (1964). Charte internationale sur la conservation et la restauration des monuments et des sites (charte de Venise 1964), Venise, Art. 9 et 10.

Journal officiel de la république Algérienne. (1998). "Loi n 98-04 du 20 Safar 1419 correspondant au 15 juin 1998 relative à la protection du patrimoine culturel", J.O., 44, pp. 3-15.

Ministère de la guerre. (1842). Tableau de la situation des établissements français en Algérie en 1841, Imprimerie Royale Ed., Paris, p. 24.

Rehabimed. (2007). Méthode Rehabimed. Architecture traditionnelle Méditerranéenne. Vol. II. Réhabilitation Bâtiments, Rehabimed Ed., Barcelone, pp. 297-329.

Reveyron, N. (2002). "L'apport de l'archéologie du bâti dans la monographie d'architecture", In Situ. Revue des patrimoines, 2, (online) in http://journals.openedition.org/insitu/1200 (30 April 2019). 
